\title{
BIOCHEMICAL REMOVAL OF HAP PRECURSORS FROM COAL
}

\author{
Quarterly Technical Report
}

Reporting Period: October 1, 1996 - December31, 1996

Author: Dr. Gregory J. Olson

Report Issue Date: January, 1997

DE-AC22-95PC95155

Little Bear Laboratories, Inc.

P.O. Box 1434

Red Lodge, MT 59068 
This report was prepared as an account of work sponsored by an agency of the United States Government. Neither the United States Government nor any agency thereof, nor any of their employees, makes any warranty, express or implied, or assumes any legal responsibility for the accuracy, completeness or usefulness of any information, apparatus, product or process disclosed, or represents that its use would not infringe privately owned rights. Reference herein to any specific commercial product, process, or service by trade name, trademark, manufacturer, or otherwise does not necessarily constitute or imply its endorsement, recommendation, or favoring by the United States Government or any agency thereof. The views and opinions of authors expressed herein do not necessarily state or reflect those of the United States Government or any agency thereof. 
page

Abstract 3

Executive Summary 3

Introduction 4

Results and Discussion 5

1. Shake Flask Tests--Kentucky No. 9 Coal 5

2. Completion of Indiana No. 5 Column Test 11

3. Column Tests with High Concentration Ferric 15

4. Stockton Coal Analysis 20

5. INEL Slurry Column Testing 21

$\begin{array}{lr}\text { Conclusions } & 21\end{array}$ 


\begin{abstract}
The rate of pyrite removal from Kentucky No. 9 coal was about $6 \%$ per day in a shake flask test--similar to previous rates with Indiana and Pittsburgh coal. Significant percentages of HAP precursors As, Co, Cd, Mn and Ni were removed from the coal. These same five HAP precursors also were significantly removed from Indiana No. 5 coal in a column test. Additionally, column tests were conducted with Indiana No. 5 and Pittsburgh No. 8 coal using high initial concentrations of ferric ions. These tests showed faster depyritization of coal than in previous tests done with low initial ferric ion concentrations. In addition, faster and more extensive removal of $\mathrm{Cd}, \mathrm{Co}, \mathrm{Mn}$ and $\mathrm{Ni}$ from Indiana No. 5 coal occurred under high ferric conditions. Analysis of HAP precursors in Stockton Coal, used by PETC in HAP precursor combustion-mass balance tests, was done and compared to PETC analytical data. The INEL slurry column reactor was operated in several shake down runs to prepare for complete HAP precursor removal-mass balance tests.
\end{abstract}

\title{
Executive Summary
}

This fifth quarterly report covers the period of October through December of 1996. Results are presented of pyrite and HAP precursor removal from Kentucky No. 9 coal in shake flasks and from Indiana No. 5 coal in columns. With Kentucky coal, rates of pyrite oxidation were about $6 \%$ per day, and significant As, Co, Cd, Mn and Ni were removed from the coal. These same five HAP precursors also were significantly removed from Indiana No. 5 coal. Additionally, test results are presented of pyrite and HAP precursor removal from Indiana No. 5 and Pittsburgh No. 8 coal using high initial concentrations of ferric ions. These tests showed faster depyritization of coal than in previous tests done with low initial ferric ion concentrations. In addition, faster and more extensive removal of $\mathrm{Cd}, \mathrm{Co}, \mathrm{Mn}$ and $\mathrm{Ni}$ from Indiana No. 5 coal occurred under high ferric conditions. High solution ferric ion concentrations are expected in any biodepyritization process due to progressive biooxidation of pyrite to ferric sulfate. Ferric ions are probably the primary oxidant of pyrite and many of the HAP precursors in coal. Analysis of HAP precursors in Stockton Coal, used by PETC in HAP precursor combustion-mass balance tests, was done and compared to PETC analytical data. The INEL slurry column reactor was operated in several shake down runs to prepare for complete HAP precursor removal-mass balance tests. Good separation of coal from ash-forming minerals was observed in these tests. 
This project is investigating microbiological removal of 13 hazardous air pollutant (HAP) precursors and pyrite from four U.S. coals. Shake flask and column tests are being conducted to determine the rate and extent of the process. Each test determines the rate of microbial pyrite oxidation, the extent of HAP precursor removal, and the mass balance of HAP precursor elements.

Tests run during this quarter investigated for the first time the removal of pyrite and HAP precursors in columns at high initial concentrations of ferric ions $(10 \mathrm{~g} / \mathrm{l})$. Ferric ions are probably the primary oxidant of pyrite and most HAP precursor minerals in coal. Previous shake flask and column tests were begun with coal and nutrient salts only. As microbial pyrite oxidation proceeded, ferric ion concentrations in solution slowly increased with time. However, since high concentrations of ferric ions are expected in microbial coal processing streams, it is important to determine the effects elevated ferric concentrations on HAP precursor and pyrite removal. Such highly oxidizing solutions may be more effective in removing the undesirable inorganic components of coal, as long as increased precipitation of iron, sulfate or other elements does not occur.

The Idaho National Engineering Laboratory (INEL) slurry column reactor was operated in a series of test runs this quarter in preparation for full operation of reactor performance, complete with HAP precursor analyses and mass balance determinations. The slurry column reactor employs both physical separations and microbial oxidation for removal of pyrite. Its performance in HAP precursor removal will be evaluated in the coming quarter. 


\section{Shake Flask Tests--Results of Kentucky Coal (Task 2)}

The fourth and final shake flask test was completed. This test employed Kentucky No. 9 bituminous coal, obtained from the Penn State Coal Sample Bank (sample DECS-18). The material was sieved and the minus 100 mesh fraction used in the shake flask test. As in previous tests, flasks contained $20 \%$ solids in dilute mineral salts (modified Kelly medium) adjusted to $\mathrm{pH} 2$ with sulfuric acid. Two flasks were inoculated with pyrite oxidizing bacteria. An additional two flasks served as abiotic controls and were treated with a biocidal solution of $2 \%$ thymol in methanol.

The rate of pyrite oxidation (Table 1) was calculated from the maximum slope of the iron and sulfate dissolution curves shown in Figure 1. This interval occurred from days 4 to 10 for $\mathrm{Fe}$ and days 3 to 8 for sulfate. The controls showed little if any increase in solution iron and sulfate.

Table 1. Rates of Pyrite Oxidation (\% per day) in Kentucky Coal Shake Flask Test

\begin{tabular}{|c|c|c|}
\hline flask & Based on solution $\mathrm{Fe}$ & Based on solution $\mathrm{SO}_{4}$ \\
\hline \hline cells -1 & 7.5 & 6.6 \\
\hline cells -2 & 6.3 & 4.8 \\
\hline
\end{tabular}

The rate of depyritization of Kentucky coal as estimated from solution iron and sulfate concentrations (5 to $7 \%$ per day) was similar to that found with Pittsburgh (8 to $9 \%$ per day) and Indiana (4 to $5 \%$ per day) coals (second quarter report).

The analysis of the raw Kentucky feed coal had not been completed at the end of the quarter. However, the Penn State coal bank value for pyritic sulfur in this coal is $2.37 \%$ and this value was used in the above plots. The analysis of the recovered coal residues (Table 2) indicated approximately $94 \%$ biodepyritization, based on the $2.37 \%$ pyritic sulfur value, confirming the rapid and extensive biodepyritization of Kentucky coal. Solution sulfate and iron concentrations indicated about $75 \%$ of the pyrite was removed through microbial activity. These data indicated some precipitation of iron and sulfate occurred.

Significant amounts of HAP precursor elements As, Co, Cd, Mn, and Ni were removed from Kentucky No. 9 coal based on an analysis of raw coal compared recovered residues (Table 2). These 5 HAP precursors were similarly removed in previous shake flask tests with Pittsburgh and Indiana coals. Solution analyses (Table 3) confirmed the removal of these elements from the coal, and also indicated some removal of selenium and lead. Selenium was not significantly removed by microbial activity in previous shake flask tests with other coals. 
Figure 1. Kinetics of Depyritization of Kentucky No. 9 Coal; Shake Flask
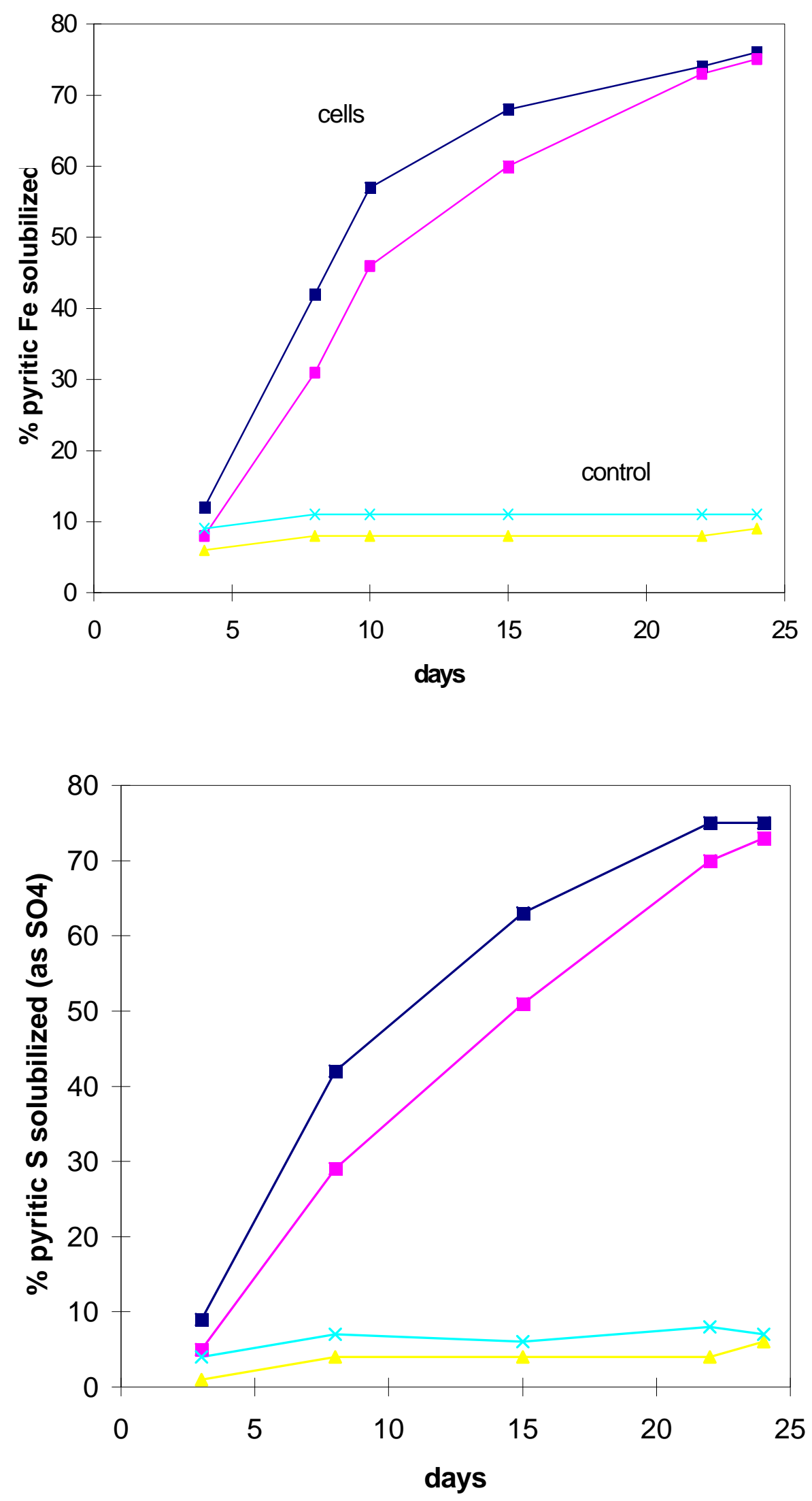
Intermediate solutions in the Kentucky coal shake flask test (Table 3) indicated that As and $\mathrm{Cd}$ increased in concentration with time as the extent of pyrite oxidation increased. There was a much higher concentration of these elements in solutions of inoculated flasks compared to uninoculated flasks. Cadmium extractions of $137 \%$ and $134 \%$ (Table 3) are high probably due to analytical error in determining the $\mathrm{Cd}$ content of the starting coal which was only slightly above the detection limit. Solutions in inoculated flasks also contained significantly higher concentrations of $\mathrm{Se}$ and $\mathrm{Pb}$. These data suggest biooxidation played an important role in the extent of dissolution of $\mathrm{As}$, Cd, Se, and $\mathrm{Pb}$. The concentrations of $\mathrm{Se}$ and $\mathrm{Pb}$ increased little after 10 days. Additionally, $\mathrm{Mn}, \mathrm{Co}$ and $\mathrm{Ni}$ increased relatively little after the initial analysis at day 10. However, differences in solution concentration of these elements between inoculated and control flasks were less pronounced than with $\mathrm{As}, \mathrm{Cd}, \mathrm{Se}$, and $\mathrm{Pb}$. This suggests a portion of $\mathrm{Mn}, \mathrm{Co}$ and $\mathrm{Ni}$ were solubilized by acidic conditions in the flasks.

Mass balances for HAP precursors in Kentucky coal were largely within a range of 93 to $120 \%$ (Table 4). Values outside this range were associated with $\mathrm{Hg}$ and $\mathrm{Cd}$ which were present in the coal at near detection limits. 
Table 2. Analysis of Kentucky Coal, Shake Flask Test (-100 mesh, dry basis)

\begin{tabular}{|c|c|c|c|c|c|}
\hline & Raw coal & Biotreated & Control & \%loss-microbial & \% loss-control \\
\hline \% carbon & $\begin{array}{c}\text { not yet } \\
\text { done }\end{array}$ & $\begin{array}{l}70.99 \\
70.89 \\
\end{array}$ & $\begin{array}{l}68.85 \\
68.20 \\
\end{array}$ & & \\
\hline$\%$ hydrogen & & $\begin{array}{l}4.52 \\
4.51 \\
\end{array}$ & $\begin{array}{l}4.33 \\
4.57 \\
\end{array}$ & & \\
\hline$\%$ nitrogen & & $\begin{array}{l}1.44 \\
1.45\end{array}$ & $\begin{array}{l}1.39 \\
1.38\end{array}$ & & \\
\hline$\%$ sulfur & & $\begin{array}{l}.25 \\
3.22 \\
\end{array}$ & $\begin{array}{l}4.77 \\
4.59 \\
\end{array}$ & & \\
\hline$\%$ ash & & $\begin{array}{l}12.15 \\
11.92\end{array}$ & $\begin{array}{l}13.82 \\
14.03\end{array}$ & & \\
\hline $\begin{array}{l}\% \text { oxygen } \\
\text { (difference) }\end{array}$ & & $\begin{array}{l}7.65 \\
8.01\end{array}$ & $\begin{array}{l}6.84 \\
7.23\end{array}$ & & \\
\hline Btu/lb & & $\begin{array}{l}12422 \\
12471\end{array}$ & $\begin{array}{l}12243 \\
12245\end{array}$ & & \\
\hline \% pyritic S & & $\begin{array}{l}0.13 \\
0.17 \\
\end{array}$ & $\begin{array}{l}1.68 \\
1.95 \\
\end{array}$ & & \\
\hline \% sulfate S & & $\begin{array}{l}1.08 \\
1.06 \\
\end{array}$ & $\begin{array}{l}1.01 \\
0.96 \\
\end{array}$ & & \\
\hline $\begin{array}{l}\text { \% organic S } \\
\text { (difference) }\end{array}$ & & $\begin{array}{l}2.04 \\
1.99\end{array}$ & $\begin{array}{l}2.08 \\
1.68 \\
\end{array}$ & & \\
\hline $\begin{array}{l}\text { HAPs, ug/g } \\
\text { coal }\end{array}$ & & & & & \\
\hline mercury & 0.06 & $\begin{array}{l}0.08 \\
0.09\end{array}$ & $\begin{array}{l}0.08 \\
0.07\end{array}$ & $\begin{array}{l}0 \\
0\end{array}$ & $\begin{array}{l}0 \\
0\end{array}$ \\
\hline arsenic & 8.6 & $\begin{array}{l}4.6 \\
4.6\end{array}$ & $\begin{array}{l}9.0 \\
8.4\end{array}$ & $\begin{array}{l}47 \\
47\end{array}$ & $\begin{array}{l}0 \\
2\end{array}$ \\
\hline cadmium & 0.3 & $\begin{array}{l}<0.1 \\
<0.1\end{array}$ & 0.1 & $\begin{array}{l}>67 \\
>67 \\
\end{array}$ & 67 \\
\hline chromium & 9.7 & $\begin{array}{l}9.4 \\
9.9 \\
\end{array}$ & $\begin{array}{l}9.9 \\
9.7 \\
\end{array}$ & $\begin{array}{l}3 \\
0 \\
\end{array}$ & $\begin{array}{l}0 \\
0 \\
\end{array}$ \\
\hline selenium & 1.1 & $\begin{array}{l}0.9 \\
0.9\end{array}$ & $\begin{array}{l}1.1 \\
1.0\end{array}$ & $\begin{array}{l}31 \\
31\end{array}$ & $\begin{array}{l}15 \\
23\end{array}$ \\
\hline antimony & $<0.9$ & $\begin{array}{l}<0.9 \\
<0.9 \\
\end{array}$ & $\begin{array}{l}<0.9 \\
<0.9 \\
\end{array}$ & $\begin{array}{l}0 \\
0 \\
\end{array}$ & $\begin{array}{l}0 \\
0 \\
\end{array}$ \\
\hline beryllium & 0.4 & $\begin{array}{l}0.4 \\
0.4 \\
\end{array}$ & $\begin{array}{l}0.4 \\
0.4 \\
\end{array}$ & $\begin{array}{l}0 \\
0 \\
\end{array}$ & $\begin{array}{l}0 \\
0 \\
\end{array}$ \\
\hline cobalt & 2.7 & $\begin{array}{l}1.4 \\
1.9\end{array}$ & $\begin{array}{l}1.8 \\
2.2\end{array}$ & $\begin{array}{l}48 \\
30\end{array}$ & $\begin{array}{l}33 \\
19\end{array}$ \\
\hline lead & 11 & $\begin{array}{l}9.8 \\
8.9 \\
\end{array}$ & $\begin{array}{l}9.5 \\
9.4 \\
\end{array}$ & $\begin{array}{l}11 \\
19 \\
\end{array}$ & $\begin{array}{l}14 \\
15\end{array}$ \\
\hline manganese & 74 & $\begin{array}{l}16 \\
15\end{array}$ & $\begin{array}{l}19 \\
18\end{array}$ & $\begin{array}{l}78 \\
80\end{array}$ & $\begin{array}{l}74 \\
76\end{array}$ \\
\hline nickel & 7.8 & $\begin{array}{l}4.0 \\
4.1\end{array}$ & $\begin{array}{l}5.9 \\
6.5\end{array}$ & $\begin{array}{l}49 \\
47\end{array}$ & $\begin{array}{l}24 \\
17\end{array}$ \\
\hline chlorine (\%) & $\begin{array}{c}\text { not yet } \\
\text { done }\end{array}$ & $\begin{array}{l}840 \\
810 \\
\end{array}$ & $\begin{array}{l}780 \\
820 \\
\end{array}$ & & \\
\hline fluorine & $\begin{array}{c}\text { not yet } \\
\text { done }\end{array}$ & $\begin{array}{l}50 \\
50 \\
\end{array}$ & $\begin{array}{l}50 \\
50 \\
\end{array}$ & & \\
\hline
\end{tabular}


Table 3. \% of Coal HAP Precursors in Leach Solutions in Kentucky Shake Flasks

\begin{tabular}{|c|c|c|}
\hline & Day 10 & Final (day 24) \\
\hline $\begin{array}{l}\text { \% pyrite biooxidation } \\
\text { (based on solution Iron } \\
\text { concentration) }\end{array}$ & $\begin{array}{c}57 \text { (cells) } \\
46 \text { (cells) } \\
8 \text { (control) } \\
11 \text { (control) }\end{array}$ & $\begin{array}{c}76 \\
75 \\
9 \\
11\end{array}$ \\
\hline Arsenic & $\begin{array}{c}29 \\
24 \\
2 \\
2\end{array}$ & $\begin{array}{c}43 \\
43 \\
3 \\
3\end{array}$ \\
\hline Cadmium & $\begin{array}{l}77 \\
76 \\
25 \\
29 \\
\end{array}$ & $\begin{array}{c}137 \\
134 \\
43 \\
38 \\
\end{array}$ \\
\hline Chromium & $\begin{array}{l}3 \\
3 \\
3 \\
3 \\
\end{array}$ & $\begin{array}{l}<1 \\
<1 \\
<1 \\
<1\end{array}$ \\
\hline Selenium & $\begin{array}{l}14 \\
12 \\
<1 \\
2\end{array}$ & $\begin{array}{l}13 \\
13 \\
<1 \\
<1\end{array}$ \\
\hline Beryllium & $\begin{array}{l}4 \\
5 \\
4 \\
4 \\
\end{array}$ & $\begin{array}{l}<1 \\
<1 \\
<1 \\
<1\end{array}$ \\
\hline Cobalt & $\begin{array}{l}51 \\
51 \\
37 \\
36 \\
\end{array}$ & $\begin{array}{l}56 \\
48 \\
35 \\
39 \\
\end{array}$ \\
\hline Lead & $\begin{array}{c}20 \\
13 \\
5 \\
3 \\
\end{array}$ & $\begin{array}{c}17 \\
12 \\
8 \\
2 \\
\end{array}$ \\
\hline Manganese & $\begin{array}{l}87 \\
88 \\
75 \\
74\end{array}$ & $\begin{array}{l}76 \\
74 \\
74 \\
74\end{array}$ \\
\hline Nickel & $\begin{array}{l}41 \\
38 \\
21 \\
23\end{array}$ & $\begin{array}{l}49 \\
47 \\
22 \\
24\end{array}$ \\
\hline
\end{tabular}

$\mathrm{Hg}, \mathrm{Sb}, \mathrm{F}$ and $\mathrm{Cl}$ were not solubilized and are not shown above. The \% extraction was calculated by dividing the mass of HAP precursor in solution by the mass of HAP precursor in starting coal (data are shown in the first two columns of Table 4). 
Table 4. Mass Balances for Kentucky Coal Shake Flask Test*

\begin{tabular}{|c|c|c|c|c|}
\hline & $\begin{array}{c}\text { starting coal } \\
\text { mg }\end{array}$ & $\begin{array}{c}\text { solution } \\
\text { mg }\end{array}$ & $\begin{array}{c}\text { final coal } \\
\text { mg }\end{array}$ & $\begin{array}{c}\% \\
\text { recovery }\end{array}$ \\
\hline $\begin{array}{ll}\mathrm{Hg} & \text { cells } \\
& \text { cells } \\
& \text { control } \\
& \text { control } \\
& \end{array}$ & 0.0021 & $\begin{array}{l}<0.0001 \\
<0.0001 \\
<0.0001 \\
<0.0001\end{array}$ & $\begin{array}{l}0.0027 \\
0.0031 \\
0.0030 \\
0.0025\end{array}$ & $\begin{array}{l}129 \\
148 \\
143 \\
119\end{array}$ \\
\hline arsenic & 0.301 & $\begin{array}{l}0.129 \\
0.129 \\
0.008 \\
0.007\end{array}$ & $\begin{array}{l}0.161 \\
0.161 \\
0.315 \\
0.294\end{array}$ & $\begin{array}{l}96 \\
96 \\
107 \\
100\end{array}$ \\
\hline cadmium & 0.011 & $\begin{array}{l}0.014 \\
0.013 \\
0.004 \\
0.004 \\
\end{array}$ & $\begin{array}{l}<0.002 \\
<0.002 \\
0.004 \\
0.011 \\
\end{array}$ & \begin{tabular}{c|}
127 \\
118 \\
73 \\
136 \\
\end{tabular} \\
\hline chromium & 0.340 & $\begin{array}{l}0.001 \\
0.001 \\
0.001 \\
0.001 \\
\end{array}$ & $\begin{array}{l}0.329 \\
0.347 \\
0.347 \\
0.340 \\
\end{array}$ & $\begin{array}{l}97 \\
102 \\
102 \\
100 \\
\end{array}$ \\
\hline selenium & 0.039 & $\begin{array}{c}0.005 \\
0.005 \\
<0.001 \\
<0.001 \\
\end{array}$ & $\begin{array}{l}0.033 \\
0.032 \\
0.039 \\
0.036 \\
\end{array}$ & $\begin{array}{c}97 \\
95 \\
100 \\
93 \\
\end{array}$ \\
\hline antimony & $<0.018$ & $<0.001$ (all) & $<0.018$ (all) & -- \\
\hline beryllium & 0.014 & $\begin{array}{l}<0.001 \\
<0.001 \\
<0.001 \\
<0.001\end{array}$ & $\begin{array}{l}0.015 \\
0.015 \\
0.014 \\
0.014 \\
\end{array}$ & $\begin{array}{l}107 \\
107 \\
100 \\
100 \\
\end{array}$ \\
\hline cobalt & 0.095 & $\begin{array}{l}0.053 \\
0.046 \\
0.033 \\
0.037 \\
\end{array}$ & $\begin{array}{l}0.049 \\
0.068 \\
0.062 \\
0.076 \\
\end{array}$ & $\begin{array}{l}107 \\
120 \\
100 \\
119 \\
\end{array}$ \\
\hline lead & 0.368 & $\begin{array}{l}0.062 \\
0.045 \\
0.031 \\
0.007 \\
\end{array}$ & $\begin{array}{l}0.342 \\
0.311 \\
0.331 \\
0.329 \\
\end{array}$ & $\begin{array}{c}109 \\
97 \\
98 \\
91 \\
\end{array}$ \\
\hline manganese & 2.583 & $\begin{array}{l}1.958 \\
1.901 \\
1.922 \\
1.908\end{array}$ & $\begin{array}{l}0.550 \\
0.525 \\
0.648 \\
0.613\end{array}$ & $\begin{array}{c}97 \\
94 \\
100 \\
98\end{array}$ \\
\hline nickel & 0.273 & $\begin{array}{l}0.140 \\
0.144 \\
0.207 \\
0.228 \\
\end{array}$ & $\begin{array}{l}0.140 \\
0.144 \\
0.207 \\
0.228 \\
\end{array}$ & $\begin{array}{c}100 \\
100 \\
98 \\
107 \\
\end{array}$ \\
\hline fluorine & not yet done & & & \\
\hline chlorine & not yet done & & & \\
\hline
\end{tabular}

${ }^{*}$ Four values appear for each HAP precursor. As shown for $\mathrm{Hg}$ above, the first two rows are for the two inoculated flasks, the last two rows are for control flasks. Mass balances were determined by adding the total $\mathrm{mg}$ in solution ( $\mathrm{mg} / \mathrm{l} \times$ liters) to the $\mathrm{mg}$ recovered in coal residues $(\mathrm{mg} / \mathrm{kg} \times \mathrm{kg}$ ) divided by the $\mathrm{mg}$ in raw coal $(\mathrm{mg} / \mathrm{kg} \times \mathrm{kg})$. 
In the previous quarterly report, the kinetics of pyrite dissolution from Indiana No. 5 coal and concentrations of HAP precursors in leach solutions were reported. Tables 5 , 6 , and 7 below report the analysis of coal residues, the concentration of HAP precursors in the final leach solutions, and the mass balance calculations for this column test.

Based on the pyritic $S$ analyses of the residues, the Indiana No. 5 coal was 71 to $74 \%$ biodepyritized compared to $38 \%$ depyritization in control columns. However, the organic sulfur content of biotreated and control coals increased by about $0.5 \%$ on an absolute basis. Only about $0.2 \%$ of the $0.5 \%$ increase was due to sulfate. Some accumulation of elemental sulfur may have occurred in this coal. Only about $50 \%$ biodepyritization of the coal was indicated from solution iron and sulfate concentrations (see 4th quarter report, fig. 2).

Residue and solution analyses indicated that significant amounts of HAP precursors As, Cd, Co, Mn, and Ni were removed from coal by microbial leaching. Loss of $\mathrm{Hg}$, present at near detection limits, was suggested by residue analysis but was not confirmed by solution data (Table 7). Slight removal of Se also occurred.

As and $\mathrm{Cd}$ tended to increase in concentration in solution with time, tracking pyrite oxidation as estimated from solution $\mathrm{Fe}$ and sulfate (Table 6). In addition, significantly higher concentrations of these elements occurred in inoculated columns. In contrast, $\mathrm{Co}, \mathrm{Mn}$, and $\mathrm{Ni}$ increased little after 16 days. Concentrations of these elements were similar in inoculated and control columns. These data suggest 1) Co, Mn, and Ni were largely mobilized by acidic conditions, and 2) As and Cd were mobilized largely due to biooxidation. 
Table 5. Analysis of Indiana No 5 Coal, Column Test ( $28 \times 100$ mesh, dry basis).

\begin{tabular}{|c|c|c|c|c|c|}
\hline & Raw coal & Biotreated & Control & \%loss-microbial & \% loss-control \\
\hline$\%$ carbon & 72.92 & $\begin{array}{l}74.09 \\
74.20 \\
\end{array}$ & 73.22 & & \\
\hline \% hydrogen & 5.03 & $\begin{array}{l}5.13 \\
5.09\end{array}$ & 4.96 & & \\
\hline$\%$ nitrogen & 1.54 & $\begin{array}{l}1.57 \\
1.58 \\
\end{array}$ & 1.55 & & \\
\hline$\%$ sulfur & 3.69 & $\begin{array}{l}3.03 \\
2.98 \\
\end{array}$ & 3.64 & $\begin{array}{l}18 \\
19\end{array}$ & 1 \\
\hline$\%$ ash & 9.44 & $\begin{array}{l}7.17 \\
7.11 \\
\end{array}$ & 8.10 & $\begin{array}{l}24 \\
25 \\
\end{array}$ & 14 \\
\hline $\begin{array}{l}\text { \% oxygen } \\
\text { (difference) }\end{array}$ & 7.38 & $\begin{array}{l}9.01 \\
9.04\end{array}$ & 8.53 & & \\
\hline Btu/lb & 13180 & $\begin{array}{l}13138 \\
13151 \\
\end{array}$ & 13068 & & \\
\hline \% pyritic S & 1.92 & $\begin{array}{l}0.56 \\
0.49 \\
\end{array}$ & 1.19 & $\begin{array}{l}71 \\
74 \\
\end{array}$ & 38 \\
\hline \% sulfate S & 0.07 & $\begin{array}{l}0.25 \\
0.19 \\
\end{array}$ & 0.19 & & \\
\hline $\begin{array}{l}\text { \% organic S } \\
\text { (difference) }\end{array}$ & 1.70 & $\begin{array}{l}2.22 \\
2.30\end{array}$ & 2.26 & & \\
\hline \multicolumn{6}{|l|}{$\begin{array}{l}\text { HAPs, ug/g } \\
\text { coal }\end{array}$} \\
\hline mercury & 0.11 & $\begin{array}{l}0.08 \\
0.08 \\
\end{array}$ & 0.09 & $\begin{array}{l}27 \\
27 \\
\end{array}$ & 18 \\
\hline arsenic & 4.1 & $\begin{array}{l}2.9 \\
3.1 \\
\end{array}$ & 3.7 & $\begin{array}{l}29 \\
24 \\
\end{array}$ & 10 \\
\hline cadmium & 0.6 & $\begin{array}{l}<0.1 \\
<0.1\end{array}$ & 0.1 & $\begin{array}{l}>83 \\
>83 \\
\end{array}$ & 83 \\
\hline chromium & 12 & $\begin{array}{l}13 \\
13 \\
\end{array}$ & 12 & $\begin{array}{l}0 \\
0 \\
\end{array}$ & 0 \\
\hline selenium & 2.0 & $\begin{array}{l}0.9 \\
1.3 \\
\end{array}$ & 1.0 & $\begin{array}{l}55 \\
35 \\
\end{array}$ & 50 \\
\hline antimony & $\begin{array}{l}<1.0 \\
<1.0 \\
\end{array}$ & $\begin{array}{l}<1.0 \\
<1.0 \\
\end{array}$ & $<1.0$ & $\begin{array}{l}0 \\
0 \\
\end{array}$ & 0 \\
\hline beryllium & 1.3 & $\begin{array}{l}1.5 \\
1.4\end{array}$ & 1.4 & $\begin{array}{l}0 \\
0\end{array}$ & 0 \\
\hline cobalt & 4.3 & $\begin{array}{l}1.8 \\
1.9 \\
\end{array}$ & 2.3 & $\begin{array}{l}58 \\
56 \\
\end{array}$ & 47 \\
\hline lead & 7.7 & $\begin{array}{l}7.9 \\
7.8 \\
\end{array}$ & 7.4 & $\begin{array}{l}0 \\
0\end{array}$ & 0 \\
\hline manganese & 30 & $\begin{array}{l}9 \\
9 \\
\end{array}$ & 12 & $\begin{array}{l}70 \\
70 \\
\end{array}$ & 60 \\
\hline nickel & $\begin{array}{l}15 \\
15 \\
\end{array}$ & $\begin{array}{l}10 \\
10 \\
\end{array}$ & 12 & $\begin{array}{l}33 \\
33 \\
\end{array}$ & 20 \\
\hline chlorine (\%) & 0.01 & $\begin{array}{l}<0.01 \\
<0.01 \\
\end{array}$ & $<0.01$ & -- & -- \\
\hline fluorine & 63 & $\begin{array}{l}40 \\
40 \\
\end{array}$ & 40 & $\begin{array}{l}36 \\
36 \\
\end{array}$ & 36 \\
\hline
\end{tabular}


Table 6. $\%$ of Coal HAP Precursors in Leach Solutions ${ }^{1}$ in Indiana No. 5 Columns

\begin{tabular}{|c|c|c|c|}
\hline & Day 16 & Day 34 & Final - Day 54 \\
\hline \multirow{3}{*}{$\begin{array}{l}\text { \% pyrite biooxidation } \\
\text { (based on solution } \mathrm{SO}_{4} \\
\text { concentration) }\end{array}$} & 29 (cells) & 48 & 45 \\
\hline & 30 (cells) & 47 & 50 \\
\hline & 15 (control) & 22 & 31 \\
\hline \multirow[t]{3}{*}{ Arsenic } & 17 & 32 & 32 \\
\hline & 20 & 25 & 29 \\
\hline & 4 & 14 & 21 \\
\hline \multirow[t]{3}{*}{ Cadmium } & 50 & 66 & 66 \\
\hline & 43 & 49 & 51 \\
\hline & 17 & 28 & 36 \\
\hline \multirow[t]{3}{*}{ Selenium } & 5 & 9 & 8 \\
\hline & 6 & 7 & 7 \\
\hline & 1 & 3 & 4 \\
\hline \multirow[t]{3}{*}{ Beryllium } & 2 & 2 & 2 \\
\hline & 2 & 2 & 1 \\
\hline & 2 & 2 & 1 \\
\hline \multirow[t]{3}{*}{ Cobalt } & 41 & 40 & 37 \\
\hline & 41 & 32 & 32 \\
\hline & 37 & 35 & 37 \\
\hline \multirow[t]{3}{*}{ Lead } & 3 & 3 & 7 \\
\hline & 4 & 2 & 6 \\
\hline & 5 & 2 & 6 \\
\hline \multirow[t]{3}{*}{ Manganese } & 46 & 45 & 41 \\
\hline & 45 & 38 & 36 \\
\hline & 43 & 38 & 40 \\
\hline \multirow[t]{3}{*}{ Nickel } & 24 & 27 & 28 \\
\hline & 23 & 23 & 26 \\
\hline & 19 & 18 & 25 \\
\hline
\end{tabular}

${ }^{1}$ Less than $1 \%$ of $\mathrm{Sb}, \mathrm{Hg}, \mathrm{Cl}, \mathrm{Cr}$ and $\mathrm{F}$ was solubilized. 
Table 7. Mass Balances for Indiana No. 5 Coal Column Test*

\begin{tabular}{|c|c|c|c|c|}
\hline & $\begin{array}{c}\text { starting coal } \\
\mathrm{mg}\end{array}$ & $\begin{array}{c}\text { solution } \\
\mathrm{mg}\end{array}$ & $\begin{array}{c}\text { final coal } \\
\mathrm{mg}\end{array}$ & $\%$ recovery \\
\hline $\begin{array}{ll}\mathrm{Hg} & \begin{array}{l}\text { control } \\
\text { cells } \\
\text { cells }\end{array} \\
\end{array}$ & 0.022 & $\begin{array}{l}\text { ND } \\
\text { ND } \\
\text { ND } \\
\end{array}$ & $\begin{array}{l}0.018 \\
0.017 \\
0.017 \\
\end{array}$ & $\begin{array}{l}82 \\
77 \\
77 \\
\end{array}$ \\
\hline arsenic & 0.82 & $\begin{array}{l}0.16 \\
0.24 \\
0.21\end{array}$ & $\begin{array}{l}0.74 \\
0.58 \\
0.62\end{array}$ & $\begin{array}{l}110 \\
100 \\
101\end{array}$ \\
\hline cadmium & 0.12 & $\begin{array}{l}0.04 \\
0.08 \\
0.06 \\
\end{array}$ & $\begin{array}{l}0.03 \\
\text { ND } \\
\text { ND }\end{array}$ & $\begin{array}{l}59 \\
66 \\
51\end{array}$ \\
\hline chromium & 2.34 & $\begin{array}{l}\text { ND } \\
\text { ND } \\
\text { ND }\end{array}$ & $\begin{array}{l}2.44 \\
2.60 \\
2.56 \\
\end{array}$ & $\begin{array}{l}104 \\
111 \\
109 \\
\end{array}$ \\
\hline selenium & 0.40 & $\begin{array}{l}0.02 \\
0.03 \\
0.03 \\
\end{array}$ & $\begin{array}{l}0.21 \\
0.17 \\
0.26\end{array}$ & $\begin{array}{l}58 \\
50 \\
73\end{array}$ \\
\hline beryllium & 0.26 & $\begin{array}{l}0.004 \\
0.004 \\
0.004\end{array}$ & $\begin{array}{l}0.28 \\
0.29 \\
0.28\end{array}$ & $\begin{array}{l}110 \\
115 \\
111\end{array}$ \\
\hline cobalt & 0.86 & $\begin{array}{l}0.32 \\
0.32 \\
0.28\end{array}$ & $\begin{array}{l}0.47 \\
0.35 \\
0.38\end{array}$ & $\begin{array}{l}92 \\
77 \\
76\end{array}$ \\
\hline lead & 1.54 & $\begin{array}{l}0.09 \\
0.10 \\
0.09\end{array}$ & $\begin{array}{l}1.48 \\
1.58 \\
1.56\end{array}$ & $\begin{array}{l}102 \\
109 \\
107\end{array}$ \\
\hline manganese & 6.0 & $\begin{array}{l}2.4 \\
2.4 \\
2.2 \\
\end{array}$ & $\begin{array}{l}2.5 \\
1.8 \\
1.7 \\
\end{array}$ & $\begin{array}{l}82 \\
71 \\
65 \\
\end{array}$ \\
\hline nickel & 3.0 & $\begin{array}{l}0.76 \\
0.84 \\
0.77\end{array}$ & $\begin{array}{l}2.4 \\
2.0 \\
1.9 \\
\end{array}$ & $\begin{array}{c}104 \\
93 \\
90 \\
\end{array}$ \\
\hline chlorine & 10 & $\begin{array}{l}<5 \\
<5 \\
<5\end{array}$ & $\begin{array}{l}<10 \\
<10 \\
<10\end{array}$ & \\
\hline fluorine & 6.3 & $\begin{array}{l}<0.01 \\
<0.01 \\
<0.01\end{array}$ & $\begin{array}{l}4.0 \\
4.0 \\
4.0 \\
\end{array}$ & \\
\hline
\end{tabular}

*Three values are shown for each HAP precursor. As shown for $\mathrm{Hg}$ above, the first two rows in each box are for the two inoculated columns, the last row is for the control columns. $\mathrm{Cl}$ and $\mathrm{F}$ data not yet available 
3. Column Tests--Leaching of Pittsburgh No. 8 and Indiana No. 5 Coal with High Concentration Ferric Solutions

The purpose of these tests was to determine the rate and extent of pyrite and HAP precursor removal from coal in columns by solutions initially containing high ferric ion concentration. Results will be compared to data from previous column tests where the initial ferric ion concentration was zero. The high concentration of ferric ions might be more effective in removing HAP precursors from coal.

Tests at high initial ferric ion concentration are more representative of conditions that would exist in a process for biological pyrite and HAP precursor removal from coal, where fresh coal would be leached with solution from coal previously treated with pyrite oxidizing bacteria. Microbial oxidation of pyrite in the fresh coal would result in progressively higher concentrations of ferric ions (and other solutes) in leach solutions. Eventually, solutions would have to be treated (neutralized) to remove acidity, iron, and HAP precursors.

Two reservoirs each containing $200 \mathrm{ml}$ of MKM solution (dilute mineral satls at $\mathrm{pH} 2$ to support the growth of pyrite oxidizing bacteria) plus $10 \mathrm{~g} / \mathrm{l}$ of ferrous iron (as ferrous sulfate heptahydrate) were inoculated with a mixed microbial population pregrown in a slurry of MKM plus pyrite and Pittsburgh 8 coal. The reservoirs were aerated. After 6 days, the iron oxidizing bacteria oxidized $99 \%$ of the ferrous iron to ferric iron and the redox potential in the solutions had risen from $593 \mathrm{mV}$ (SHE) to 732 to $742 \mathrm{mV}$. At this point, the solution from the reservoirs was recirculated either through a column containing $100 \mathrm{~g}$ of $28 \times 100$ mesh Indiana No. 5 or Pittsburgh No. 8 coal. Recirculation continued for 23 days at room temperature. Samples were removed periodically for determination of $\mathrm{pH}$, redox potential, ferrous and total iron, sulfate, and HAP precursors.

Tables 8, 910 , and 11 show the analysis of feed coal and biooxidized residues, \% extraction of HAP precursors, and mass balances for Pittsburgh and Indiana coals.

Neither solution iron or sulfate concentrations provided a good estimate of pyrite biooxidation rate. Acid consumption by the coals early in the solution recirculation process probably resulted in some precipitation of these analytes. Indeed, rust colored precipitates were observed in reservoirs and in columns, and the sulfate-S content of the biooxidized residues was higher than the raw coal. By analysis of biooxidized residues, 59 and $50 \%$ depyritization occurred over the 23 days for Pittsburgh and Indiana coals, respectively (Table 8 ). This corresponds to a pyrite oxidation rate over $2 \%$ per day, which is about twice the rate observed with these coals in previous column tests (see third quarter report, Table 9). This higher rate was probably due to maintenance of higher concentrations of ferric ions in the reservoir solutions than occurred in previous tests. Despite the precipitation of iron, the solution ferric iron concentrations throughout the test were 4 to $10 \mathrm{~g} / \mathrm{l}$, and the redox potentials were $>700$ 
$\mathrm{mV}$ (SHE). In the previous column tests, the ferric ion concentrations reached 4 to $5 \mathrm{~g}$ per liter only near the conclusion of the tests.

Residue analyses suggested significant amounts (>20\%) of $\mathrm{Be}, \mathrm{Co}, \mathrm{Mn}$ and Ni were removed from Pittsburgh coal by the high ferric solutions. Significant $\mathrm{Cd}$, Se, Co, Pb, and $\mathrm{Mn}$ were reoved from Indiana coal. The solution analyses (Table 9) indicated Co, $\mathrm{Mn}$ and $\mathrm{Ni}$ were significantly removed from these coals in the first 17 days. Cd was also significantly removed from Indiana No. 5 coal, through less rapidly than $\mathrm{Co}, \mathrm{Mn}$, and $\mathrm{Ni}$. In contrast to past results, As removal from these coals by high ferric solutions was modest. The arsenic may have been solubilized but then precipitated along with the iron as ferric arsenate. This precipitation would have implications for coal processing and waste treatment. An acidic rinse of coal prior to application of solutions containing high ferric concentrations might be required.

The removal of $\mathrm{Co}, \mathrm{Mn}$ and $\mathrm{Ni}$ from Pittsburgh coal by high initial ferric ion concentration was more rapid than in the previous column test with low initial ferric ion concentration. The faster removal was associated with the faster removal of pyrite, since the percentage of HAP precursor removed at the end of the test $(59 \%$ pyrite oxidation) was fairly similar to the percentage of HAP precursor removed at the end of the previous test at $64-69 \%$ pyrite removal (third quarter report).

With Indiana coal, $\mathrm{Cd}, \mathrm{Co}, \mathrm{Mn}$ and $\mathrm{Ni}$ were removed more rapidly in the high ferric test (Table 9) than in the previous test (Table 6 of this report). However, the extent of HAP precursor removal was somewhat higher in the high ferric system at the end of the test (day 23 with high ferric, day 54 with low ferric) than found previously with initial low ferric concentration.

Mass balances in the high ferric test were generally good (80 to $120 \%$ ). Be and $\mathrm{Mn}$ recovery was low in Pittsburgh coal. Lead recovery was high in Indiana coal. 
Table 8. Analysis of Coal, High Ferric Column Test (+100 mesh, dry basis)

\begin{tabular}{|l||c|c|c||c|c|c|}
\hline & $\begin{array}{l}\text { Pitt. 8 } \\
\text { Raw }\end{array}$ & $\begin{array}{l}\text { Pitt. } \\
\text { Biotreated }\end{array}$ & $\begin{array}{l}\text { \% loss } \\
\text { microbial }\end{array}$ & $\begin{array}{l}\text { Ind 5 } \\
\text { Raw }\end{array}$ & $\begin{array}{l}\text { Ind 5 } \\
\text { Biotreated }\end{array}$ & $\begin{array}{l}\text { \% loss } \\
\text { microbial }\end{array}$ \\
\hline \hline \% carbon & 64.02 & 64.42 & & 72.92 & 71.85 & \\
\hline$\%$ hydrogen & 4.52 & 4.60 & & 5.03 & 5.20 & \\
\hline$\%$ nitrogen & 1.15 & 1.18 & & 1.54 & 1.51 & \\
\hline$\%$ sulfur & 3.52 & 2.97 & 16 & 3.69 & 3.44 & 7 \\
\hline$\%$ ash & 19.46 & 19.16 & 2 & 9.44 & 8.16 & 14 \\
\hline $\begin{array}{l}\text { \% oxygen } \\
\text { (difference) }\end{array}$ & 7.33 & 7.67 & & 7.38 & 9.84 & \\
\hline Btu/lb & 11649 & 11687 & & 13180 & 12970 & \\
\hline$\%$ pyritic S & 1.44 & 0.59 & 59 & 1.92 & 0.96 & 50 \\
\hline$\%$ sulfate S & 0.32 & 0.61 & & 0.07 & 0.30 & \\
\hline $\begin{array}{l}\% \text { organic S } \\
\text { (difference) }\end{array}$ & 1.76 & 1.77 & & 1.70 & 2.18 & \\
\hline \hline $\begin{array}{l}\text { HAPs, ug/g } \\
\text { coal }\end{array}$ & & & & & & \\
\hline mercury & 0.11 & 0.12 & 0 & 0.04 & 0.08 & 0 \\
\hline arsenic & 9.3 & 8.3 & 11 & 4.1 & 3.9 & 5 \\
\hline cadmium & 0.2 & $<0.1$ & $>50$ & 0.7 & 0.2 & 71 \\
\hline chromium & 44 & 36 & 18 & 12 & 14 & 0 \\
\hline selenium & 1.8 & 1.9 & 0 & 1.8 & 1.2 & 33 \\
\hline antimony & 0.6 & 0.6 & 0 & 0.9 & 1.1 & 0 \\
\hline beryllium & 0.7 & 0.4 & 43 & 1.3 & 1.2 & 8 \\
\hline cobalt & 6.4 & 3.6 & 44 & 4.3 & 1.9 & 56 \\
\hline lead & $<1$ & $<1$ & -- & 7.4 & $<1$ & $>87$ \\
\hline manganese & 450 & 88 & 80 & 30 & 12 & 60 \\
\hline nickel & 20 & 10 & 50 & 15 & 13 & 13 \\
\hline fluorine & 120 & 110 & 8 & 55 & 50 & 9 \\
\hline
\end{tabular}


Table 9. \% of Coal HAP Precursors in Leach Solutions in Pittsburgh No. 8 and Indiana No. 5 High Ferric Columns

\begin{tabular}{|l|c|c||c|c|}
\hline & $\begin{array}{l}\text { Day 11 } \\
\text { Pitt. No. 8 }\end{array}$ & $\begin{array}{l}\text { Final (day 23) } \\
\text { Pitt. No. 8 }\end{array}$ & $\begin{array}{l}\text { Day 11 } \\
\text { Ind. No. 5 }\end{array}$ & $\begin{array}{l}\text { Final (day 23) } \\
\text { Ind. No. 5 }\end{array}$ \\
\hline \hline $\begin{array}{l}\text { \% pyrite } \\
\text { oxidation }\end{array}$ & -- & $59^{1}$ & -- & $50^{1}$ \\
\hline arsenic & 6 & 10 & 13 & 13 \\
\hline cadmium & 83 & 100 & 42 & 87 \\
\hline selenium & 3 & 5 & 8 & 10 \\
\hline beryllium & 9 & 9 & 2 & 2 \\
\hline cobalt & 35 & 40 & 56 & 54 \\
\hline manganese & 45 & 49 & 87 & 79 \\
\hline nickel & 36 & 41 & 40 & 33 \\
\hline
\end{tabular}

${ }^{1}$ pyrite concentrations based on residue analysis

Table 10. Mass Balances for Pittsburgh Coal High Ferric Column Test

\begin{tabular}{|l|c|c|c|c|}
\hline & starting coal mg & solution $\mathrm{mg}$ & final coal $\mathrm{mg}$ & $\%$ recovery \\
\hline \hline mercury & 0.011 & $<0.001$ & 0.012 & 109 \\
\hline arsenic & 0.93 & 0.10 & 0.83 & 100 \\
\hline cadmium & 0.02 & 0.02 & $<0.02$ & -- \\
\hline chromium & 4.41 & 0.23 & 3.60 & 87 \\
\hline selenium & 0.18 & 0.01 & 0.19 & 100 \\
\hline antimony & 0.06 & $<0.01$ & 0.06 & 103 \\
\hline beryllium & 0.07 & 0.01 & 0.04 & 71 \\
\hline cobalt & 0.64 & 0.26 & 0.36 & 96 \\
\hline lead & $<0.10$ & 0.01 & $<0.10$ & -- \\
\hline manganese & 45 & 22 & 8.8 & 68 \\
\hline nickel & 1.87 & 0.78 & 0.99 & 94 \\
\hline chlorine & not yet done & & & \\
\hline fluorine & not yet done & & & \\
\hline
\end{tabular}


Table 11. Mass Balances for Indiana Coal High Ferric Column Test

\begin{tabular}{|c|c|c|c|c|}
\hline & $\begin{array}{l}\text { starting coal } \\
\text { mg }\end{array}$ & $\begin{array}{l}\text { solution } \\
\text { mg }\end{array}$ & $\begin{array}{l}\text { final coal } \\
\mathrm{mg}\end{array}$ & $\%$ recovery \\
\hline cells & 0.011 & ND & 0.008 & 80 \\
\hline arsenic & 0.46 & 0.06 & 0.39 & 97 \\
\hline cadmium & 0.07 & 0.06 & 0.02 & 114 \\
\hline chromium & 1.17 & 0.02 & 1.36 & 117 \\
\hline selenium & 0.15 & 0.015 & 0.12 & 90 \\
\hline antimony & 0.09 & ND & 0.09 & 100 \\
\hline beryllium & 0.13 & 0.002 & 0.12 & 94 \\
\hline cobalt & 0.43 & 0.23 & 0.19 & 98 \\
\hline lead & 0.11 & 0.07 & 0.12 & 173 \\
\hline manganese & 3.0 & 2.36 & 1.24 & 120 \\
\hline nickel & 1.5 & 0.50 & 1.34 & 123 \\
\hline chlorine & & & & \\
\hline fluorine & & & & \\
\hline
\end{tabular}




\section{Analysis of Stockton Coal for HAP Precursors}

A PETC in house research project studied mass balances of HAP precursors associated with combustion of Stockton (Kentucky) coal. In order to compare analytical results conducted by PETC with those at LBL, we analyzed the raw coal (ground to minus 60 mesh) for its content of HAP precursors (Table 12).

Table 12. HAP precursors in Stockton coal

\begin{tabular}{|c|c|c|c|}
\hline element & ug/g coal & $\begin{array}{c}\text { \% spike } \\
\text { recovery }\end{array}$ & $\begin{array}{c}\text { PETC } \\
\text { value }\end{array}$ \\
\hline \hline $\mathrm{Hg}$ & 0.04 & 55 & 0.095 \\
& 0.04 & & 0.085 \\
\hline $\mathrm{As}$ & 5.7 & 84 & 2.4 \\
& 5.2 & & 1.5 \\
\hline $\mathrm{Cd}$ & $<0.2$ & 91 & 0.67 \\
& $<0.2$ & & $<2.0$ \\
\hline $\mathrm{Cr}$ & 44 & 106 & 40 \\
& 48 & & 27 \\
\hline $\mathrm{Se}$ & 2.5 & 68 & 5.0 \\
& 3.1 & & 5.0 \\
\hline $\mathrm{Sb}$ & $<0.5$ & 80 & 0.90 \\
& $<0.5$ & & $<0.57$ \\
\hline $\mathrm{Be}$ & 1.5 & 90 & 2.0 \\
& 1.5 & & 1.7 \\
\hline $\mathrm{Co}$ & 8.2 & 97 & 8.3 \\
& 9.0 & & 11 \\
\hline $\mathrm{Pb}$ & 4.4 & 97 & 6.5 \\
& 2.0 & & 7.2 \\
\hline $\mathrm{Mn}$ & 476 & 102 & 250 \\
& 459 & & 5.9 \\
\hline $\mathrm{Ni}$ & 21 & 94 & 24 \\
& 18 & & 27 \\
\hline $\mathrm{ash} \%$ & 14.1 & & 12.7 \\
& & & 12.1 \\
\hline
\end{tabular}

PETC values on as received basis, LBL on dry basis

PETC values are for coal from pilot unit (top value) and full scale (bottom value).

The two LBL values are results of complete duplicate ashing, digestion and analysis. The spike was a third sample carried through all treatments except the low temperature ashing. With some elements, considerable variation occurred between LBL andf PETC values. In the case of $\mathrm{Hg}$ and $\mathrm{Se}$, the lower $\mathrm{LBL}$ values are reflected by low spike recoveries. In the case of $\mathrm{Mn}$, considerable variation in the PETC value in coal from pilot and full scale tests was observed. 


\section{INEL Slurry Column Test Work (Task 4)}

Initial reactor tests were conducted at the Idaho National Engineering Laboratory with Pittsburgh coal and a consortium of bacteria provided by Little Bear Labs. The iron oxidizing bacteria were grown in an electrolytic cell (converting $\mathrm{Fe}^{3+}$ to $\mathrm{Fe}^{2+}$ ) to increase cell numbers. Coal was added to the slurry column reactor to make a $35 \% \mathrm{w} / \mathrm{w}$ slurry. Each reactor was inoculated with 3 liters of a cell/coal suspension.

Four batches of coal were processed in preparation for complete tests involving HAP precursor measurements and mass balances. The first run lasted 41 days and was designed primarily to allow bacteria to adapt to the coal. The next 3 runs were to check operating and sampling procedures. Jeff Richards of Unifield Engineering (subcontractors) was on hand for a few days to observe reactor performance and design mass balance procedures.

Good physical separation of ash forming minerals from coal occurred in 3 of the 4 runs (Table 13). There were some problems in closing water and coal balances with a new washing procedure due to excessive coal fines.

Table 13. Coal-Ash Separations in Test Runs of Slurry Column Reactor at INEL

\begin{tabular}{|c|c|c|c|}
\hline Run \# & Days & \% Ash in Product Coal & \% Ash in Waste Coal \\
\hline \hline 1 & 41 & 12.2 & 43.8 \\
\hline 2 & 19 & 11.6 & 46.1 \\
\hline 3 & 10 & 12.9 & 25.4 \\
\hline 4 & 17 & 15.7 & 53.0 \\
\hline
\end{tabular}

During the coming quarter, retention time will be cut back to five days and these batches will be analyzed for HAP precursors.

\section{Conclusions}

Kentucky coal behaved similarly to Pittsburgh and Indiana coals in shake flask tests in terms of rate of depyritization and in the identity of HAP precursors removed (As, Co, $\mathrm{Cd}, \mathrm{Mn}, \mathrm{Ni}$ ). Indiana No. $5 \mathrm{coal}$ in columns also was biooxidized with removal of the same HAP precursors. Pittsburgh and Indiana coals in columns with high initial ferric ion concentrations were more rapidly depyritized than in previous column tests with these coals. Certain HAP precursors in Indiana coal were removed more rapidly in the high initial ferric ion tests. The INEL slurry column reactor was tested for coal-ash separations and for sampling protocols. The reactor is now ready for complete testing of coal biodepyritization and HAP precursor removal. 